\title{
Exercise training has greater effects on insulin sensitivity in daughters of patients with type 2 diabetes than in women with no family history of diabetes
}

\author{
N. D. Barwell • D. Malkova • C. N. Moran • \\ S. J. Cleland • C. J. Packard • V. A. Zammit • \\ J. M. R. Gill
}

Received: 23 April 2008 / Accepted: 20 June 2008 / Published online: 29 July 2008

(C) The Author(s) 2008

\begin{abstract}
Aims/hypothesis Sedentary offspring of patients with type 2 diabetes are often more insulin-resistant than persons with no family history of diabetes, but when active or fit offspring of type 2 diabetic patients are compared with non-diabetic persons, differences in insulin resistance are less evident. This study aimed to determine the effects of an exercise training intervention on insulin sensitivity in both groups. Methods Women offspring $(n=34)$ of type 2 diabetic patients (offspring age $35.6 \pm 7.0$ years, BMI $28.1 \pm 5.1 \mathrm{~kg} / \mathrm{m}^{2}$ ) and 36

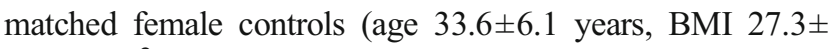
$4.7 \mathrm{~kg} / \mathrm{m}^{2}$ ) participated. Body composition, fitness and metabolic measurements were made at baseline and after a controlled 7 week exercise intervention.
\end{abstract}

Electronic supplementary material The online version of this article (doi:10.1007/s00125-008-1097-6) contains supplementary material, which is available to authorised users.

N. D. Barwell • D. Malkova • C. N. Moran · J. M. R. Gill ( $\triangle)$ Institute of Diet, Exercise and Lifestyle (IDEAL),

Faculty of Biomedical and Life Sciences, West Medical Building,

University of Glasgow, University Avenue,

Glasgow G12 8QQ, UK

e-mail: j.gill@bio.gla.ac.uk

D. Malkova

Division of Developmental Medicine,

University of Glasgow, Glasgow, UK

\section{S. J. Cleland · C. J. Packard}

Division of Cardiovascular and Medical Sciences,

University of Glasgow, Glasgow, UK

\section{A. Zammit}

Clinical Sciences Research Institute, Warwick Medical School, University of Warwick, Warwick, UK
Results At baseline, insulin sensitivity index (ISI) was $22 \%$ lower in offspring than controls $(p<0.05)$, despite similar body fat and maximal oxygen uptake $\left(\dot{V} \mathrm{O}_{2 \max }\right)$ values in the two groups. ISI increased by $23 \%(p<0.05)$ in offspring following the exercise intervention, compared with $7 \%$ (NS) in the controls. Increases in $\dot{V} \mathrm{O}_{2 \max }$ were similar in both groups (controls $12 \%$, offspring $15 \%, p<0.05$ for both). Plasma leptin concentrations decreased significantly in the offspring $(-24 \%, p<0.01)$ but not in controls $(0 \%$, NS). Change in ISI correlated significantly with baseline ISI $(r=-0.47, p<0.0005)$ and change in leptin $(r=-0.43, p<$ $0.0005)$. The latter relationship was not attenuated by adjustment for changes in body fat.

Conclusions/interpretation Offspring, but not controls, significantly increased ISI in response to an exercise intervention, indicating that insulin sensitivity is more highly modulated by physical activity in daughters of patients with type 2 diabetes than in women with no family history of the disease.

\section{Trial registration: NCT00268541}

Funding: British Heart Foundation (PG/03/145).

Keywords Diabetes family history $\cdot$ Exercise $\cdot$ Insulin resistance $\cdot$ Insulin sensitivity · Leptin · Offspring · Training · Women
Abbreviations
ISI insulin sensitivity index
$\dot{V} \mathrm{O}_{2} \quad$ oxygen uptake
$\dot{V} \mathrm{O}_{2 \max }$ maximal oxygen uptake 


\section{Introduction}

The rapid increase in the prevalence of type 2 diabetes over recent years [1] must be attributed to changes in environmental factors, such as body fatness and exercise habits, as the human genome has not changed over this short period [2]. It has been suggested that being overweight with an abdominal fat distribution probably accounts for 80 to $90 \%$ [3] and a sedentary lifestyle accounts for at least $25 \%$ [4] of all type 2 diabetes incidence. However, as not all overweight and sedentary individuals develop the disease, it is clear that other non-environmental factors also play an important role in determining risk for type 2 diabetes. In sedentary populations, first-degree relatives of patients with type 2 diabetes have about three times the risk of developing diabetes than their counterparts with no family history of diabetes $[5,6]$ and, even when matched for BMI, are often more insulin-resistant than control participants [7-9], suggesting that relatives have an innate susceptibility (probably due to some combination of genetic, epigenetic and/or 'early-origins' factors) to insulin resistance and diabetes. However, in epidemiological observations, the excess risk of developing diabetes that is associated with a diabetes family history is much greater in sedentary population groups than in physically active population groups, suggesting that the increased risk associated with a diabetes family history may be diminished (although not eliminated) at high levels of physical activity [10, 11]. In addition, recent cross-sectional reports suggest that modulation of insulin resistance in offspring of patients with type 2 diabetes may be particularly amenable to habitual physical activity or cardiorespiratory fitness level, such that inactive or unfit offspring are insulin-resistant compared with control participants with no diabetes family history, whereas active or fit offspring exhibit levels of insulin sensitivity that are much closer to persons with no diabetes family history [12-14]. Taken together, these observations suggest that healthy offspring of patients with type 2 diabetes possess an innate predisposition to insulin resistance, with consequent increased diabetes risk, which manifests when sedentary. However, this appears to be highly modulated by level of physical activity. We therefore hypothesised that, in response to the same exercise training intervention, offspring of type 2 diabetes patients would improve insulin sensitivity to a greater extent than control participants with no diabetes family history.

\section{Methods}

Participants and recruitment Inclusion criteria for this trial were: female sex, in good general health, pre-menopausal with a regular menstrual cycle, non-smoker, age 20 to
45 years, sedentary lifestyle (less than $1 \mathrm{~h}$ of planned physical activity per week and a sedentary job), fasting plasma glucose $<7 \mathrm{mmol} / \mathrm{l}$, blood pressure $<160 / 90 \mathrm{mmHg}$ and either: at least one parent diagnosed with type 2 diabetes before the age of 65 years (offspring group) or no first- or second-degree relative with type 2 diabetes (control group). A total of 241 women responded to our call for volunteers via newspaper articles, a study website, posters, the University newsletter and personal contacts. Of these, 76 attended for screening and 70 women (34 offspring and 36 controls) fulfilled the inclusion criteria and agreed to participate in the trial (for participant flow diagram, see Electronic supplementary material [ESM] Fig. 1). Offspring and control groups were matched for age and BMI. Three volunteers were taking prescribed medication (selective serotonin reuptake inhibitors in two participants, 1-thyroxine in one participant) and medication was constant throughout their participation in the study. No participants reported initiation of prescription medications during the study. All participants gave written informed consent prior to inclusion in this trial which was approved by the Research Ethics Committee of the North Glasgow University Hospitals National Health Service Trust.

Study design Participants were randomised into Exercise or Delayed exercise intervention arms using a computer program. The study design is shown in Fig. 1. The Exercise intervention group immediately commenced a progressive 7 week exercise intervention following baseline testing (OGTT, fitness and body composition) and underwent postintervention testing on completion of this programme. The post-intervention OGTT was performed 15 to $24 \mathrm{~h}$ after participants' final exercise session. The Delayed exercise intervention group underwent a 7 week control period of continuing their normal lifestyle following baseline testing. Baseline testing was then repeated, before these participants underwent the same 7 week exercise intervention as the Exercise intervention group, with post-intervention testing on completion of the intervention. Thus, participants were studied at 8-week intervals to allow, as far as possible, for all metabolic, fitness and body composition assessments for

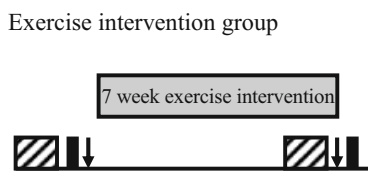

Delayed exercise intervention group

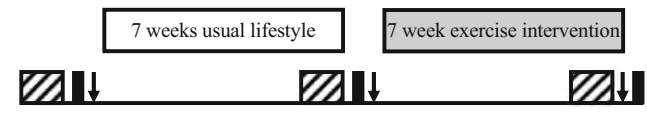

Fig. 1 Study design. Hatched boxes, 7 day dietary assessment; black boxes, OGTT; arrows, fitness and body composition assessment 
each individual to be made in the same menstrual cycle phase [15]. Participants were asked not to alter their dietary habits during their participation in the study and completed 7 day weighed food diaries prior to each metabolic testing day. Diaries were analysed using a computerised version of food composition tables (CompEat Pro; Nutrition Systems, Banbury, UK).

Fitness testing Participants performed an incremental, submaximal treadmill walking test to determine lactate threshold [16] and estimate maximal oxygen uptake $\left(\dot{V} \mathrm{O}_{2 \max }\right)$ [17]. Treadmill speed was set at $5 \mathrm{~km} / \mathrm{h}$ with gradient increasing by $2 \%$ per $5 \mathrm{~min}$ stage. Expired air samples for determination of oxygen uptake $\left(\dot{V} \mathrm{O}_{2}\right)$ and carbon dioxide production, as well as heart rates were taken throughout. Capillary blood samples were taken at the end of each stage and blood lactate concentrations were determined using an analyser (Analox GM7; Analox Instruments, Hammersmith, UK). Tests were terminated once participants achieved $\sim 85 \%$ of their age-predicted maximum heart rate and the $\dot{V} \mathrm{O}_{2}$ vs heart rate relationship was then extrapolated to age-predicted maximum (220-age [years]) to estimate $\dot{V} \mathrm{O}_{2 \max }$.

Oral glucose tolerance test Participants reported to the metabolic suite after $12 \mathrm{~h}$ overnight fast. A cannula was introduced into an antecubital vein and after a $10 \mathrm{~min}$ interval, a fasting blood sample was taken. Participants then underwent an OGTT, consuming a $75 \mathrm{~g}$ oral glucose load in $300 \mathrm{ml}$ liquid, with further blood samples taken at $30 \mathrm{~min}$ intervals for $120 \mathrm{~min}$.

Body composition assessment Dual x-ray absorptiometry scans (Lunar Prodigy DEXA; GE Healthcare Diagnostic Imaging, Slough, UK) were used to determine body composition and fat distribution. Height, body mass, and waist and hip circumferences were also determined using standard protocols [18].

Exercise intervention Participants underwent a 7 week endurance-type exercise training programme starting with $3 \times 30 \mathrm{~min}$ of exercise in the first week and building progressively to $5 \times 60 \mathrm{~min}$ of exercise in weeks 6 and 7 of the intervention. All participants were given free access to the University Sports Centre, provided with a downloadable heart rate monitor (Polar s610i, Polar Electro Oy, Kempele, Finland) and instructed to exercise at $65-80 \%$ of their predicted maximum heart rate for all exercise sessions. Participants were allowed to use whichever cardiovascular exercise equipment they preferred (e.g. treadmill, stepper, cycle ergometer, rowing ergometer) or attend scheduled aerobic exercise classes. Alternatively, participants were allowed to run, cycle or perform other modes of exercise at other locations, if they preferred, provided they completed the required duration and intensity of exercise. One exercise session per week was supervised by an investigator. At this session, heart rate data from the previous week's sessions were downloaded to verify compliance and the exercise plan for the following week was agreed. Other than participating in the exercise intervention, participants were requested to make no changes to their lifestyle for the duration of the study.

Analytical methods Blood samples were collected into potassium EDTA tubes and placed on ice. All samples were separated within $15 \mathrm{~min}$ of collection and stored at $-80^{\circ} \mathrm{C}$ until analysis. Plasma total cholesterol and HDL-cholesterol were determined according to the Lipid Research Clinics Program Manual of Laboratory Operations [19]. LDLcholesterol was calculated using the Friedewald equation. Enzymatic colorimetric methods using commercially available kits were used to determine triacylglycerol (Roche Diagnostics, Mannheim, Germany), glucose (Roche Diagnostics), NEFA (Wako Chemicals, Neuss, Germany) and 3-hydroxybutyrate (Randox Laboratories, Crumlin, UK) concentrations. Insulin was determined using a commercially available ELISA with $<0.01 \%$ cross-reactivity with proinsulin (Mercodia, Uppsala, Sweden). Leptin, which was measured because it influences insulin sensitivity [20], has been reported to change with exercise training [21] and may differ between control and offspring groups [22] as defined here, was determined using a commercially available ELISA ( $\mathrm{R}$ and D Systems, Abingdon, UK). All samples for each participant were analysed in a single analyser run. Coefficients of variation were $<3.1 \%$ for all non-ELISA assays, $<4 \%$ for the insulin ELISA and $8 \%$ for the leptin ELISA.

Calculation of insulin sensitivity Insulin sensitivity was calculated from fasting and post-glucose plasma glucose and insulin concentrations using the insulin sensitivity index (ISI) devised by Matsuda and DeFronzo [23], i.e. 10,000/ $\sqrt{ }[$ (fasting glucose $\times$ fasting insulin) $\times$ (mean glucose during OGTT $\times$ mean insulin during OGTT)] (with glucose in $\mathrm{mg} / \mathrm{dl}$ and insulin in $\mu \mathrm{U} / \mathrm{ml}$ ). Mean glucose and insulin concentrations during OGTT were calculated as the average of the $30,60,90$ and $120 \mathrm{~min}$ values. This index is highly correlated with the rate of whole-body glucose disposal during a euglycaemic-hyperinsulinaemic clamp [23]. Based on data from the two baseline measurements made in the participants randomised to the Delayed exercise intervention arm, the within-participant test-retest coefficient of variation for ISI was $12.2 \%$, with mean values for ISI differing by $3.2 \%$ between the two baseline measurements.

Statistical analysis Data were analysed using Statistica (version 6.0; StatSoft, Tulsa, OK, USA) and Minitab 
(version 13.1; Minitab, State College, PA, USA). Prior to analysis, data were tested for normality using the Anderson-Darling normality test and, if necessary, logarithmically transformed. We calculated $2 \mathrm{~h}$ AUCs following glucose ingestion using the trapezium rule. Differences between offspring and control groups at baseline were determined using unpaired $t$ tests. In participants randomised to the Delayed exercise group, the second baseline measurements were used for the baseline assessments, unless the participant dropped out between the first and second baselines measures $(n=5)$, in which case the first baseline values were used. Changes from the assessment conducted immediately pre-intervention to that conducted post-intervention in all participants who completed the intervention (i.e. between the first and second sets of tests in participants randomised to the 'Exercise intervention and between the second and third sets of tests in participants randomised to Delayed exercise intervention) were compared by two-way ANOVA (group $\times$ trial) with repeated measures on the 'trial' factor. The group $\times$ trial interaction term was used to determine whether the offspring and control participants responded differently to the intervention, with post hoc Tukey tests used to identify which group(s) changes occurred in. In separate analyses, similar comparisons were made between the first and second sets of tests in participants randomised to the Delayed exercise intervention to determine whether a 'study effect' could have contributed to the observed changes. Associations between variables were assessed using Pearson product-moment correlations. Multiple regression analyses were performed to establish the independence of relationships.

\section{Results}

Baseline data Physical and metabolic characteristics and energy intakes of the participants at baseline are shown in Table 1 . The offspring group had $27 \%$ higher fasting insulin concentrations $(p<0.05)$ and $22 \%$ lower ISI $(p<0.05)$ than controls. There were no significant differences between control and offspring participants in any of the other measured variables. Macronutrient compositions of diets also did not differ significantly between groups (data not shown).

Compliance to exercise intervention Six offspring participants and two control participants dropped out of the study before completion of the exercise intervention. Reasons for their withdrawal are shown on the flow diagram (ESM Fig. 1). Of participants who completed the intervention and attended for post-intervention testing, control participants $(n=34)$ completed $28 \pm 6$ exercise sessions (out of 32
Table 1 Physical variables at baseline in control $(n=36)$ and offspring participants $(n=34)$

\begin{tabular}{|c|c|c|}
\hline Variable & Controls & Offspring \\
\hline Age (years) & $33.6 \pm 6.1$ & $35.6 \pm 7.0$ \\
\hline Body mass (kg) & $73.0 \pm 14.8$ & $76.6 \pm 15.1$ \\
\hline BMI $\left(\mathrm{kg} / \mathrm{m}^{2}\right)$ & $27.3 \pm 4.7$ & $28.1 \pm 5.1$ \\
\hline Body fat $(\%)$ & $39.6 \pm 6.4$ & $41.4 \pm 6.6$ \\
\hline Total fat mass $(\mathrm{kg})$ & $28.9 \pm 10.1$ & $31.3 \pm 10.2$ \\
\hline Trunk fat mass $(\mathrm{kg})$ & $14.2 \pm 5.3$ & $16.3 \pm 5.6$ \\
\hline Total lean mass (kg) & $42.1 \pm 5.6$ & $42.6 \pm 6.0$ \\
\hline Waist circumference $(\mathrm{cm})$ & $84.4 \pm 11.6$ & $89.1 \pm 13.1$ \\
\hline Hip circumference $(\mathrm{cm})$ & $106.7 \pm 9.7$ & $109.0 \pm 10.1$ \\
\hline Systolic blood pressure (mmHg) & $117.4 \pm 12.7$ & $120.3 \pm 14.3$ \\
\hline Diastolic blood pressure (mmHg) & $72.1 \pm 8.9$ & $76.4 \pm 11.1$ \\
\hline $\begin{array}{l}\text { Energy intake ( } \mathrm{kJ}[\mathrm{kg} \text { body mass }]^{-1} \\
\left.\text { day }^{-1}\right)\end{array}$ & $108 \pm 27$ & $114 \pm 34$ \\
\hline$\dot{V} \mathrm{O}_{2 \max }$ & $32.7 \pm 6.7$ & $30.4 \pm 4.8$ \\
\hline $\begin{array}{l}\dot{V} \mathrm{O}_{2} \text { at lactate threshold } \\
(\mathrm{ml} \mathrm{kg}-1 \mathrm{~min}-1)\end{array}$ & $21.0 \pm 4.1$ & $21.0 \pm 3.8$ \\
\hline Fasting glucose $(\mathrm{mmol} / \mathrm{l})$ & $4.8 \pm 0.5$ & $4.9 \pm 0.7$ \\
\hline Fasting insulin ${ }^{\mathrm{a}}(\mathrm{pmol} / \mathrm{l})$ & $37.2 \pm 22.8$ & $47.4 \pm 27.0^{\prime}$ \\
\hline Glucose AUC $([\mathrm{mmol} / \mathrm{l}] \times \mathrm{h})$ & $12.9 \pm 2.6$ & $14.0 \pm 3.1$ \\
\hline Insulin $\mathrm{AUC}^{\mathrm{a}}([\mathrm{pmol} / \mathrm{l}] \times \mathrm{h})$ & $604 \pm 426$ & $742 \pm 466$ \\
\hline ISI $^{\mathrm{a}}$ & $7.31 \pm 3.81$ & $5.67 \pm 3.38$ \\
\hline Fasting $\mathrm{NEFA}^{\mathrm{a}}(\mathrm{mmol} / \mathrm{l})$ & $0.52 \pm 0.18$ & $0.55 \pm 0.17$ \\
\hline Fasting 3-hydroxybutyrate $(\mu \mathrm{mol} / \mathrm{l})$ & $63 \pm 48$ & $85 \pm 74$ \\
\hline Total cholesterol (mmol/l) & $4.23 \pm 1.04$ & $4.17 \pm 1.06$ \\
\hline Fasting triacylglycerol ${ }^{\mathrm{a}}(\mathrm{mmol} / \mathrm{l})$ & $0.86 \pm 0.30$ & $0.98 \pm 0.36$ \\
\hline HDL-cholesterol (mmol/1) & $1.22 \pm 0.31$ & $1.27 \pm 0.28$ \\
\hline LDL-cholesterol (mmol/l) & $2.62 \pm 0.94$ & $2.45 \pm 0.94$ \\
\hline $\operatorname{Leptin}^{\mathrm{a}}(\mathrm{ng} / \mathrm{ml})$ & $17.6 \pm 10.8$ & $22.4 \pm 15.3$ \\
\hline
\end{tabular}

Values are mean $\pm \mathrm{SD}$

${ }^{\text {a }}$ Statistical analysis performed on logarithmically transformed data. ISI was calculated from fasting and post-glucose plasma and insulin concentrations according to the model of Matsuda and DeFronzo [23] $* p<0.05$ for difference from control group

possible sessions), undertaking $1,353 \pm 58 \mathrm{~min}$ of exercise at an average heart rate of $141 \pm 2$ beats per min $(73 \pm 1 \%$ of maximum heart rate) over the 7 -week intervention period. The offspring participants $(n=28)$ completed $29 \pm 7$ exercise sessions, undertaking $1,451 \pm 107 \mathrm{~min}$ of exercise at an average heart rate of $144 \pm 2$ beats per min $(74 \pm 1 \%$ of maximum heart rate). None of these values differed significantly between the two groups.

Post-intervention data Changes in physical and metabolic characteristics and energy intakes of the control and offspring groups are presented in Table 2 and Figs 2, 3, 4. $\dot{V} \mathrm{O}_{2 \max }(12 \%$ in controls, $15 \%$ in offspring, $p<0.01$ for both) and $\dot{V} \mathrm{O}_{2}$ at lactate threshold (14\% in controls, $11 \%$ in offspring, $p<0.01$ for both) increased to a similar extent in both groups. The improvement in cardiorespiratory fitness in both groups with the intervention is also illustrated by the right-shift in the $\dot{V} \mathrm{O}_{2}$ vs heart rate relationship during 

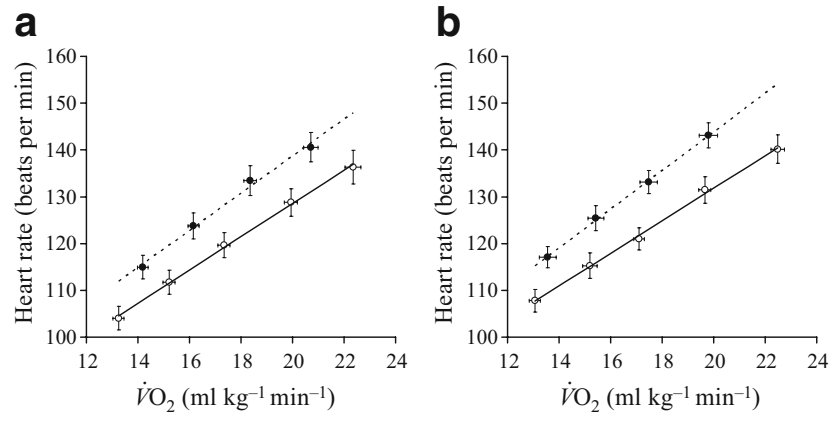

Fig. 2 Relationship between heart rate and $\dot{V} \mathrm{O}_{2}$ during incremental treadmill walking in control (a) and offspring (b) participants before (black circles) and after (white circles) the exercise intervention

Table 2 Changes in physical and metabolic variables following the exercise intervention in control $(n=34)$ and offspring participants $(n=$ 28)

\begin{tabular}{|c|c|c|}
\hline Variable & Controls & Offspring \\
\hline Body mass (kg) & $-0.15 \pm 0.28$ & $-1.20 \pm 0.44^{*} \dagger$ \\
\hline Total fat mass $(\mathrm{kg})$ & $-0.58 \pm 0.23$ & $-1.16 \pm 0.33^{* *}$ \\
\hline Trunk fat mass $(\mathrm{kg})$ & $-0.26 \pm 0.17$ & $-0.63 \pm 0.25^{*}$ \\
\hline Total lean mass (kg) & $0.15 \pm 0.43$ & $-0.04 \pm 0.28$ \\
\hline Waist circumference $(\mathrm{cm})$ & $-0.84 \pm 0.37$ & $-1.61 \pm 0.45^{* *}$ \\
\hline Hip circumference $(\mathrm{cm})$ & $-0.94 \pm 0.32 *$ & $-1.15 \pm 0.41^{*}$ \\
\hline $\begin{array}{l}\text { Systolic blood pressure } \\
(\mathrm{mmHg})\end{array}$ & $-1.6 \pm 1.8$ & $-3.4 \pm 2.3$ \\
\hline $\begin{array}{l}\text { Diastolic blood pressure } \\
(\mathrm{mmHg})\end{array}$ & $1.1 \pm 1.2$ & $-3.4 \pm 1.5 \dagger$ \\
\hline $\begin{array}{l}\text { Energy intake }(\mathrm{kJ} \text { [kg body } \\
\left.\text { mass }]^{-1} \text { day }^{-1}\right)\end{array}$ & $-5 \pm 3$ & $-6 \pm 5$ \\
\hline$\dot{V} \mathrm{O}_{2 \max }(\mathrm{ml} \mathrm{kg}-1 \mathrm{~min}-1)$ & $3.9 \pm 0.6^{* *}$ & $4.7 \pm 0.8 * *$ \\
\hline $\begin{array}{l}\dot{V} \mathrm{O}_{2} \text { at lactate threshold }(\mathrm{ml} \mathrm{kg} \\
-1 \text { min-1) }\end{array}$ & $2.8 \pm 0.5^{* *}$ & $2.4 \pm 0.7 * *$ \\
\hline Fasting glucose $(\mathrm{mmol} / \mathrm{l})$ & $-0.08 \pm 0.08$ & $-0.11 \pm 0.08$ \\
\hline Fasting insulin ${ }^{\mathrm{a}}(\mathrm{pmol} / \mathrm{l})$ & $-1.6 \pm 2.6$ & $-10.9 \pm 3.7^{*}$ \\
\hline Glucose AUC $([\mathrm{mmol} / \mathrm{l}] \times \mathrm{h})$ & $-0.2 \pm 0.4$ & $-0.6 \pm 0.4$ \\
\hline Insulin $\mathrm{AUC}^{\mathrm{a}}([\mathrm{pmol} / \mathrm{l}] \times \mathrm{h})$ & $-45 \pm 34$ & $-155 \pm 67 * * \dagger$ \\
\hline $\mathrm{ISI}^{\mathrm{a}}$ & $0.53 \pm 0.45$ & $1.34 \pm 0.39^{* *} \dagger$ \\
\hline Fasting NEFA ${ }^{a}(\mathrm{mmol} / \mathrm{l})$ & $-0.007 \pm 0.031$ & $-0.003 \pm 0.031$ \\
\hline $\begin{array}{l}\text { Fasting 3-hydroxybutyrate } \\
(\mu \mathrm{mol} / \mathrm{l})\end{array}$ & $8 \pm 7$ & $39 \pm 22$ \\
\hline Total cholesterol (mmol/l) & $-0.07 \pm 0.09$ & $-0.11 \pm 0.14$ \\
\hline $\begin{array}{l}\text { Fasting triacylglycerol }{ }^{\mathrm{a}} \\
(\mathrm{mmol} / \mathrm{l})\end{array}$ & $-0.05 \pm 0.04$ & $-0.11 \pm 0.08$ \\
\hline HDL-cholesterol (mmol/l) & $0.02 \pm 0.02$ & $0.04 \pm 0.05$ \\
\hline LDL-cholesterol (mmol/l) & $-0.07 \pm 0.08$ & $-0.09 \pm 0.10$ \\
\hline Leptin $^{\text {a }}(\mathrm{ng} / \mathrm{ml})$ & $0.0 \pm 0.9$ & $-5.3 \pm 1.4^{* *}+\dagger$ \\
\hline
\end{tabular}

Values are mean \pm SEM

${ }^{a}$ Statistical analysis performed on logarithmically transformed data. ISI was calculated from fasting and post-glucose plasma and insulin concentrations according to the model of Matsuda and DeFronzo [23] ${ }^{*} p<0.05,{ }^{* *} p<0.01$ for difference from baseline value within group $\dagger p<0.05, \dagger \dagger p<0.01$ for change from baseline between control and offspring groups sub-maximal exercise (Fig. 2). The offspring group had small but statistically significant reductions in body mass $(-1.6 \%, p<0.05)$, total fat mass $(-3.7 \%, p<0.01)$, trunk fat mass $(-3.0 \%, p<0.05)$, waist circumference $(-1.8 \%, p<0.01)$ and hip circumference $(-1.1 \%, p<0.05)$ following the exercise intervention, whereas in the control group, the only statistically significant anthropometric change was a small reduction in hip circumference $(-0.9 \%, p<0.05)$. There was also a significant interaction for the change in body mass with the intervention between the two groups $(p<0.05)$, indicating a significantly greater reduction in body mass in response to the intervention in the offspring group. Energy intakes (Table 2) and macronutrient compositions of diets (data not shown) did not change over the intervention in either group.

In the offspring group only, fasting insulin $(-23 \%, p<$ $0.05)$ and insulin AUC $(-21 \%, p<0.01)$ significantly decreased and ISI significantly increased $(23 \%, p<0.01)$ following the exercise intervention (Figs 3,4). Although a $7 \%$ increase in ISI was observed in the control group, this was not statistically significant and as the interaction for change in ISI (and insulin AUC) with the intervention was significant between the control and offspring groups $(p<$ $0.05)$, this indicates a significantly greater improvement in insulin sensitivity in response to the exercise intervention in the offspring group. Circulating leptin concentrations decreased by $24 \%$ in the offspring group $(p<0.01)$, but were unchanged in the control group, resulting in a significant group $\times$ intervention interaction $(p<0.01)$ (Table 2).
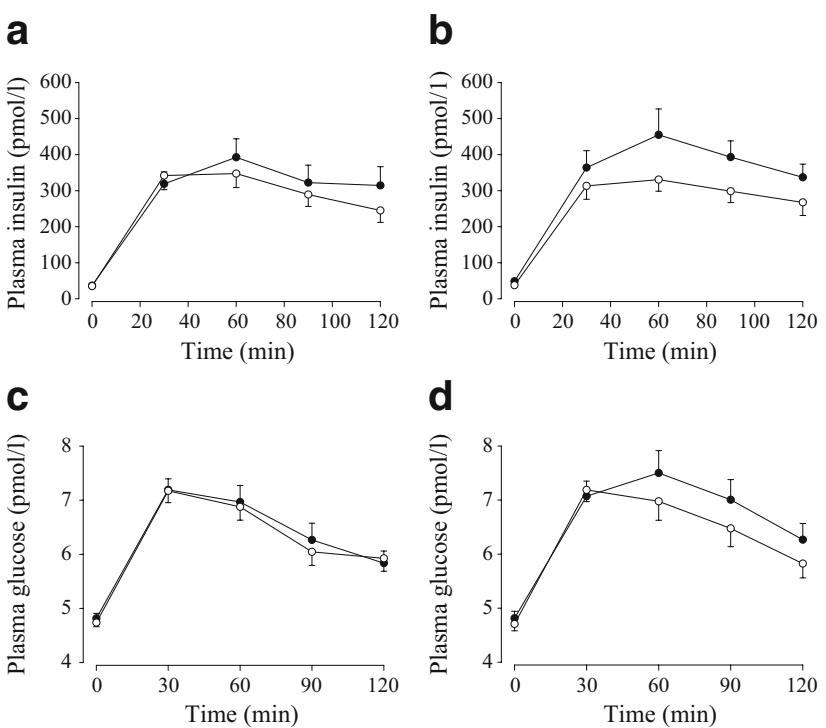

Fig. 3 Plasma insulin $(\mathbf{a}, \mathbf{b})$ and glucose $(\mathbf{c}, \mathbf{d})$ responses to a $75 \mathrm{~g}$ OGTT in control $(\mathbf{a}, \mathbf{c})$ and offspring $(\mathbf{b}, \mathbf{d})$ participants before (black circles) and after (white circles) the exercise intervention. For AUC values for these responses, see Tables 1 and 2 
a

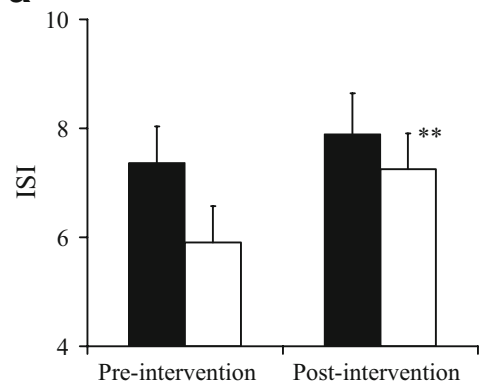

b

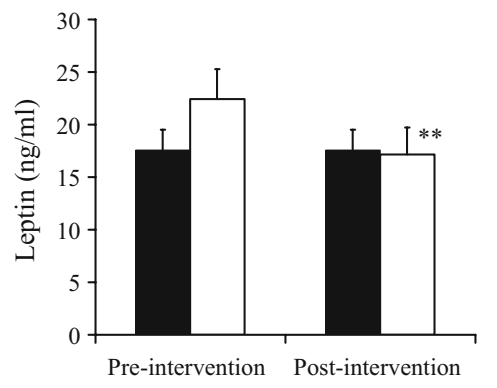

C

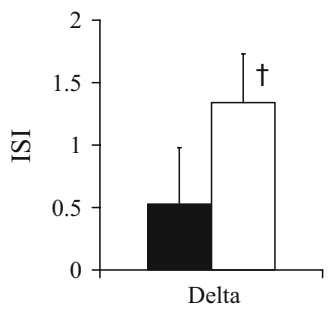

d

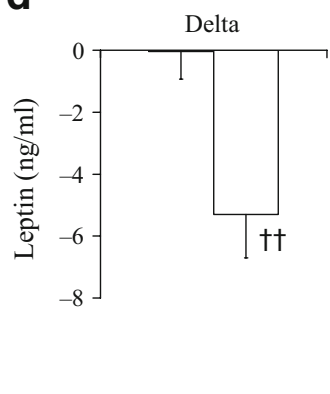

Fig. 4 Pre-intervention and post-intervention values for ISI (a) and plasma leptin (b). c, d Delta (post-intervention minus pre-intervention) values for ISI and plasma leptin concentration respectively. Black bars, control $(n=34)$; white bars, offspring $(n=28)$. Values are mean \pm SEM. Statistical analysis was performed on logarithmically transformed data. ${ }^{* *} p<0.01$ for difference from baseline value within group; $\uparrow p<0.05$ and $\dagger \dagger p<0.01$ for change from baseline between control and offspring groups

Correlations with change in ISI Change in ISI in response to the exercise intervention did not correlate significantly with changes in body fat, energy intake, $\dot{V} \mathrm{O}_{2 \max }$ or $\dot{V} \mathrm{O}_{2}$ at lactate threshold, but did correlate with baseline ISI $(r=$ $-0.47, p<0.0005)$ and with change in circulating leptin $(r=$ $-0.43, p<0.0005$ ) (Fig. 5). To determine whether the greater improvement in ISI in the offspring in response to the exercise intervention could be explained by differences in changes in fitness, energy intake, body fat or compliance

a

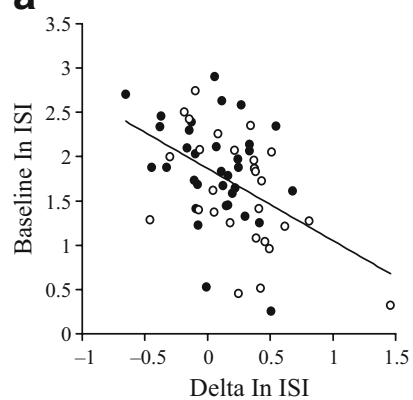

b

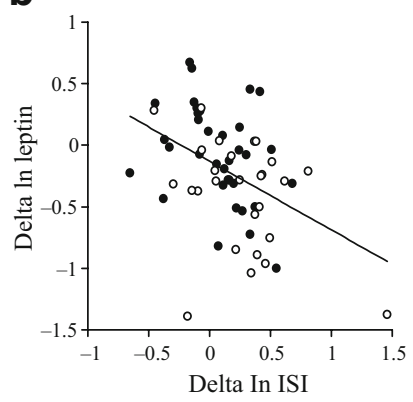

Fig. 5 Scattergrams showing relationship between (a) baseline ln ISI and change in In ISI and (b) change in ln plasma leptin concentration and change in ln ISI. Black circles, control participants; white circles, offspring. (a) $r=-0.47 ; p<0.0005$; (b) $r=-0.43$; $p<0.0005$

with the exercise intervention between the two groups, multivariate regression analyses were undertaken, with change in ISI as the response variable and group (control or offspring), together with number of minutes of exercise completed, change in body fat, change in energy intake, change in $\dot{V} O_{2 \text { max }}$ or change in $\dot{V} \mathrm{O}_{2}$ at lactate threshold as the predictor variables. In all of these analyses, group remained a significant predictor of the change in ISI in response to the intervention (all $p<0.05$ ), whereas none of the other factors included in the models were significant predictors of the change in ISI. Thus, the finding that ISI increased to a greater extent in the offspring than in the control group was not confounded by differences in compliance to the exercise intervention, differences in fat loss or differences in changes in fitness between the groups. When change in leptin concentration and group were included in a multivariate regression model, change in leptin was a significant predictor of change in ISI $(p<0.01)$, whereas the statistical significance of group was lost $(p=$ 0.29 ), indicating that the greater change in ISI in the offspring group was associated with their greater change in circulating leptin. When change in fat mass was added to this model, change in leptin remained a significant predictor of change in ISI $(p<0.0005)$. Additionally, in multivariate regression analysis including baseline ISI and group as the predictor variables, baseline ISI was a stronger significant predictor of the change in ISI $(p<0.0005)$ than group $(p=$ $0.13)$. When baseline ISI, change in leptin and group were included in the same model, baseline ISI $(p<0.0005)$ and change in leptin $(p<0.0005)$ were both independent predictors of change in ISI, whereas the significant effect of group was lost $(p=0.81)$.

Changes during the usual lifestyle period for the Delayed exercise group There were no significant changes in any of the reported physical or metabolic variables over the course of the 7-week usual lifestyle period in the control $(n=16)$ or offspring $(n=13)$ participants randomised to the Delayed exercise intervention arm and completing this aspect of the study (data not shown), except for a small, but significant, increase in lean body mass in the offspring (Pre: $44.7 \pm$ $1.7 \mathrm{~kg}$; Post: $45.6 \pm 1.8 \mathrm{~kg} ; p<0.05$ ) but not the control (Pre: $42.8 \pm 1.7 \mathrm{~kg}$; Post: $42.5 \pm 1.6 \mathrm{~kg}$; NS) groups.

\section{Discussion}

The main finding of this study was that women with a family history of type 2 diabetes significantly improved insulin sensitivity in response to an exercise training programme, whereas no significant change in insulin sensitivity was observed in women matched for age and BMI but with no diabetes family history. ISI was $22 \%$ 
lower $(p<0.05)$ in the offspring than the controls group at baseline, but only $8 \%$ lower (NS) following exercise training. This augmented response to exercise training in the offspring supports our primary hypothesis and is consistent with the literature from cross-sectional studies [12-14]. Changes in body composition over the intervention were small in both groups and although the offspring lost slightly more body fat than controls (by $\sim 0.6 \mathrm{~kg}$ ), this did not explain their greater improvement in ISI in multivariate analysis. Similarly, the larger increase in ISI in the offspring could not be explained by differences in compliance to the exercise intervention or differences in changes in fitness or energy intake between the two groups.

Despite having similar levels of adiposity and fitness, and similar dietary intakes, members of the offspring group were more insulin-resistant than the control group at baseline, consistent with other data in the literature [7-9]. It is possible that this increased baseline insulin resistance contributed to the offspring group's greater change in insulin sensitivity in response to the intervention: baseline ISI was a significant predictor of change in ISI in the present study, and other studies have reported greater exercise-induced changes in insulin sensitivity in participants who were more insulin-resistant at baseline [14, 24, 25]. As offspring of type 2 diabetes patients have an innate predisposition to insulin resistance and increased baseline insulin resistance is a well-established component of the offspring 'phenotype' [7-9], it is difficult to determine the extent to which diabetes family history versus increased baseline insulin resistance itself contributed to the increased change in ISI with exercise training in the present study. However, irrespective of whether this is due to increased insulin resistance at baseline or other underlying factors, the present data indicate that offspring of type 2 diabetes patients form an easily identifiable group who will particularly benefit from increasing their level of physical activity.

Circulating leptin concentrations decreased by $24 \%$ in response to the intervention in the offspring but were unchanged in the control group. Indeed, change in leptin was another factor that correlated significantly with change in ISI. At least part of the greater change in ISI in the offspring group could be explained by a greater change in leptin, and while it is well established that leptin concentration correlates with fat mass [20], the relationship between change in leptin and change in ISI was not attenuated by adjustment for change in fat mass. Further investigation is needed to determine why leptin changed to different degrees between the groups and whether this plays a causal role in mediating the exercise training-induced changes in ISI.

Our results differ slightly from the findings of earlier smaller studies, which found similar improvements in insulin sensitivity in response to an exercise training intervention in adults with and without a family history of type 2 diabetes [26, 27]. One possible explanation for this discrepancy is the sex of participants. The earlier reports $[26,27]$ studied mixed groups of men and women, whereas we studied women only. In the HERITAGE family study, the improvement in insulin sensitivity in response to exercise training was three times as great in men as in women (16\% vs 5\%) [28], suggesting that changes in insulin sensitivity in response to exercise training differ between the sexes. Thus, it is possible that the augmented response to exercise training in the offspring group in the present study is sex-specific and further investigation is needed to determine whether our findings extend to men.

The present investigation has a number of strengths. It is the largest study to date investigating the effects of exercise training on insulin sensitivity in offspring of patients with type 2 diabetes and thus well powered to detect changes in the main outcome measures; moreover, the exercise intervention, moreover, was tightly controlled and quantified. The control and offspring groups were well matched for age and adiposity. Although we did not strictly control diet during the intervention, we asked participants not to change their dietary habits during the study and participants undertook 'gold-standard' 7-day weighed food records during the weeks preceding each OGTT: these data indicate that dietary intakes did not differ between the groups or change over the course of the intervention. It is always difficult to undertake intervention studies in pre-menopausal women because of the potential confounding effects of menstrual cycle. However, we felt it important to study this group because they are relatively understudied in the field of cardiovascular and diabetes risk and because the development of diabetes in women has a more profound effect on cardiovascular risk than in men [29]. We attempted to control for the effects of menstrual cycle by performing pre- and post-intervention assessments at an interval of 8 weeks, so that, as far as possible, all measurements for each individual would be made in the same menstrual cycle phase [15]. Although women with very long or short menstrual cycles may not have been studied in the same phase of menstrual cycle in their pre- and postintervention tests, there is unlikely to have been a systematic difference in this respect between the groups, and the number of women this would apply to would have been small [15], so this is unlikely to have had a major influence on the study findings.

This study is not without limitations. We assessed insulin sensitivity using OGTTs, rather than 'gold standard' euglycaemic-hyperinsulinaemic clamps. The reason for this was pragmatic. Over 160 OGTTs were performed during this study and it would not have been feasible to undertake a study of this size using euglycaemic-hyperinsulinaemic clamps. However, the ISI index used in this 
study correlates well with clamp-derived measures of insulin sensitivity [23] and has been widely used in the literature (670 citations from April 2000 to March 2008, ISI Web of Knowledge, http://apps.isiknowledge.com, accessed 31 March 2008).

In conclusion, the data from the present study indicate that women with a family history of type 2 diabetes experience greater improvements in insulin sensitivity following an exercise intervention than women with no diabetes family history undertaking the same programme. Further study is needed to determine whether these findings extend to men and to elucidate the mechanisms underpinning why offspring of type 2 diabetic patients exhibited this augmented response to exercise training.

Acknowledgements This study was supported by a project grant from the British Heart Foundation (PG/03/145). The authors would like to thank C. Stewart from the University of Glasgow Sports and Recreation Service for facilitating participants' use of sports facilities for the exercise intervention. Thanks also to J. Wilson and H. Collin for technical support.

Duality of interest The authors declare that there is no duality of interest associated with this manuscript.

Open Access This article is distributed under the terms of the Creative Commons Attribution Noncommercial License which permits any noncommercial use, distribution, and reproduction in any medium, provided the original author(s) and source are credited.

\section{References}

1. Wild S, Roglic G, Green A, Sicree R, King H (2004) Global prevalence of diabetes: estimates for the year 2000 and projections for 2030. Diabetes Care 27:1047-1053

2. Booth FW, Gordon SE, Carlson CJ, Hamilton MT (2000) Waging war on modern chronic diseases: primary prevention through exercise biology. J Appl Physiol 88:774-787

3. Astrup A, Finer N (2000) Redefining type 2 diabetes: 'diabesity' or 'obesity dependent diabetes mellitus'? Obes Rev 1:57-59

4. Manson JE, Nathan DM, Krolewski AS, Stampfer MJ, Willett WC, Hennekens CH (1992) A prospective study of exercise and incidence of diabetes among US male physicians. JAMA 268:6367

5. Ohlson LO, Larsson B, Bjorntorp P et al (1988) Risk factors for type 2 (non-insulin-dependent) diabetes mellitus. Thirteen and one-half years of follow-up of the participants in a study of Swedish men born in 1913. Diabetologia 31:798-805

6. Kobberling J, Tillil H (1982) Empirical risk factors for first degree relatives of non insulin dependent diabetes. In: Kobberling J, Tattersall RB (eds) The genetics of diabetes mellitus. Academic Press, London, pp 201-209

7. Kriketos AD, Greenfield JR, Peake PW et al (2004) Inflammation, insulin resistance, and adiposity: a study of first-degree relatives of type 2 diabetic subjects. Diabetes Care 27:2033-2040

8. Nyholm B, Nielsen MF, Kristensen K et al (2004) Evidence of increased visceral obesity and reduced physical fitness in healthy insulin-resistant first-degree relatives of type 2 diabetic patients. Eur J Endocrinol 150:207-214
9. Perseghin G, Ghosh S, Gerow K, Shulman GI (1997) Metabolic defects in lean nondiabetic offspring of NIDDM parents: a crosssectional study. Diabetes 46:1001-1009

10. Hu FB, Sigal RJ, Rich-Edwards JW et al (1999) Walking compared with vigorous physical activity and risk of type 2 diabetes in women: a prospective study. JAMA 282:1433-1439

11. Sargeant LA, Wareham NJ, Khaw KT (2000) Family history of diabetes identifies a group at increased risk for the metabolic consequences of obesity and physical inactivity in EPIC-Norfolk: a population-based study. The European Prospective Investigation into Cancer. Int J Obes Relat Metab Disord 24:1333-1339

12. Higgins S, Gill JMR, Janilionyte R, Caslake MJ, Malkova D (2005) Physical activity, dietary intake and metabolic risk factors in non-diabetic daughters of patients with type II diabetes. Prev Med 40:145-151

13. Ahn CW, Song YD, Nam JH et al (2004) Insulin sensitivity in physically fit and unfit children of parents with type 2 diabetes. Diabet Med 21:59-63

14. Gill JMR, Malkova D (2006) Physical activity, fitness and cardiovascular disease risk in adults: interactions with insulin resistance and obesity. Clin Sci (Lond) 110:409-425

15. Creinin MD, Keverline S, Meyn LA (2004) How regular is regular? An analysis of menstrual cycle regularity. Contraception 70:289-292

16. Spurway NC (1992) Aerobic exercise, anaerobic exercise and the lactate threshold. Br Med Bull 48:569-591

17. American College of Sports Medicine (1995) Guidelines for exercise testing and prescription. Williams and Wilkins, Baltimore

18. Marfell-Jones M, Olds T, Stewart A, Carter L (2006) International standards for anthropometric assessment. ISAK, Potchefstroom

19. No authors listed (1975) Lipid research clinics program manual of laboratory operations. DHEW publication number (NIM) 75

20. Correia ML, Rahmouni K (2006) Role of leptin in the cardiovascular and endocrine complications of metabolic syndrome. Diabetes Obes Metab 8:603-610

21. Solomon TP, Sistrun SN, Krishnan RK et al (2008) Exercise and diet enhance fat oxidation and reduce insulin resistance in older obese adults. J Appl Physiol 104:1313-1319

22. Vauhkonen I, Niskanen L, Haffner S, Kainulainen S, Uusitupa M, Laakso M (1998) Insulin resistant phenotype is associated with high serum leptin levels in offspring of patients with non-insulindependent diabetes mellitus. Eur J Endocrinol 139:598-604

23. Matsuda M, DeFronzo RA (1999) Insulin sensitivity indices obtained from oral glucose tolerance testing: comparison with the euglycemic insulin clamp. Diabetes Care 22:1462-1470

24. Magkos F, Tsekouras Y, Kavouras SA, Mittendorfer B, Sidossis LS (2008) Improved insulin sensitivity after a single bout of exercise is curvilinearly related to exercise energy expenditure. Clin Sci (Lond) 114:59-64

25. An P, Teran-Garcia M, Rice T et al (2005) Genome-wide linkage scans for prediabetes phenotypes in response to 20 weeks of endurance exercise training in non-diabetic whites and blacks: the HERITAGE Family Study. Diabetologia 48:1142-1149

26. Perseghin G, Price TB, Petersen KF et al (1996) Increased glucose transport-phosphorylation and muscle glycogen synthesis after exercise training in insulin-resistant subjects. N Engl J Med $335: 1357-1362$

27. Ostergard T, Andersen JL, Nyholm B et al (2006) Impact of exercise training on insulin sensitivity, physical fitness, and muscle oxidative capacity in first-degree relatives of type 2 diabetic patients. Am J Physiol Endocrinol Metab 290:E998-E1005

28. Boule NG, Weisnagel SJ, Lakka TA et al (2005) Effects of exercise training on glucose homeostasis: the HERITAGE Family Study. Diabetes Care 28:108-114

29. Mak KH, Haffner SM (2003) Diabetes abolishes the gender gap in coronary heart disease. Eur Heart J 24:1385-1386 